\title{
miR-125b-5p inhibits cell proliferation by targeting ASCT2 and regulating the PI3K/AKT/mTOR pathway in an LPS-induced intestinal mucosa cell injury model
}

\author{
HUIMING GUO $^{1,2}$, JIANYUAN GAO ${ }^{2}$, YUAN QIAN $^{2}$, HUAWEI WANG $^{2}$, JIANG LIU ${ }^{2}$, \\ QINGYAN PENG ${ }^{2,3}$, YONG ZHOU ${ }^{2,3}$ and KUNHUA WANG ${ }^{2}$
}

\author{
${ }^{1}$ Department of Gynaecology, The First Affiliated Hospital of Kunming Medical University, Kunming, Yunnan 650032; \\ ${ }^{2}$ NHC Key Laboratory of Drug Addiction Medicine, Kunming Medical University, \\ Kunming, Yunnan 650500; ${ }^{3}$ The Scientific Research Laboratory Center, The First Affiliated \\ Hospital of Kunming Medical University, Kunming, Yunnan 650032, P.R. China
}

Received August 26, 2020; Accepted May 17, 2021

DOI: $10.3892 / \mathrm{etm} .2021 .10270$

\begin{abstract}
Intestinal barrier injury is an important cause of death in patients with acquired immune deficiency syndrome (AIDS). Therefore, it is of great significance to identify a therapeutic target for intestinal barrier injury to delay the progression of AIDS. microRNA (miRNA/miR)-125b-5p has an extensive role in cancer and controlling intestinal epithelial barrier function, but its role in human immunodeficiency virus-related intestinal mucosal damage remains unknown. The present study was designed to explore the effects of miR-125b-5p on lipopolysaccharide (LPS)-induced intestinal mucosal injury and the underlying mechanism. The expression of miR-125b-5p and ASCT2 mRNA was detected in colon biopsy samples of 10 patients with AIDS and 10 control healthy subjects. Human intestinal embryonic mucosa cells (CCC-HIE-2) were used to establish an LPS-induced intestinal mucosa cell injury model in vitro. Cell proliferation and apoptosis were determined by MTT assays and flow cytometry, respectively. miR-125b-5p levels and ASCT2 mRNA and protein expression levels in the LPS-induced intestinal mucosa cell injury model were detected by reverse transcription-quantitative PCR (RT-qPCR) and western blotting. The interaction between miR-125b-5p and ASCT2 was analyzed using a dual luciferase reporter assay. The results demonstrated that miR-125b-5p levels were increased and ASCT2 mRNA expression levels were decreased in colon samples from patients with AIDS and in LPS-induced intestinal mucosa cells. In the LPS-induced intestinal mucosa cell injury
\end{abstract}

Correspondence to: Dr Kunhua Wang, NHC Key Laboratory of Drug Addiction Medicine, Kunming Medical University, 1168 West Chunrong Road, Kunming, Yunnan 650500, P.R. China

E-mail: wangkunhuabio@163.com

Key words: microRNA-125b-5p, alanine serine cysteine-preferring transporter 2, lipopolysaccharide, intestinal mucosa injury, $\mathrm{PI} 3 \mathrm{~K} / \mathrm{AKT} / \mathrm{mTOR}$ pathway model, transfection with miR-125b-5p mimic inhibited cell proliferation and promoted cell apoptosis, while transfection with a miR-125b-5p inhibitor increased cell proliferation and attenuated cell apoptosis. Furthermore, miR-125b-5p mimic transfection resulted in a decrease of ASCT2 mRNA and protein expression, whereas the inhibitor increased ASCT2 mRNA and protein expression. Dual luciferase reporter assays suggested that ASCT2 was a direct target of miR-125b-5p, and its restoration weakened the effect of miR-125b-5p on LPS-induced intestinal mucosa cell injury. Transfection with the miR-125b-5p mimic also exhibited a suppressive effect on the PI3K/AKT/mTOR pathway in the LPS-induced intestinal mucosal cell injury model. Overall, the present study indicated that miR-125b-5p accelerated LPS-induced intestinal mucosa cell injury by targeting ASCT2 and upregulating the $\mathrm{PI} 3 \mathrm{~K} / \mathrm{AKT} / \mathrm{mTOR}$ pathway. The current findings may provide novel targets for the treatment of intestinal barrier injury in patients with AIDS.

\section{Introduction}

The gut is the most important organ system for metabolism and immunity, as well as a congenital barrier to prevent pathogenic bacteria and toxins and other harmful substances from passing through the intestinal tract into circulation and to ensure homeostasis of the organism $(1,2)$. At present, several studies have confirmed that the development of a variety of diseases is associated with impaired intestinal mucosal barrier, such as severe pancreatitis, sepsis, inflammatory bowel disease, diabetes, cardiovascular system diseases and acquired immune deficiency syndrome (AIDS) (3-6). Studies have demonstrated that increased intestinal epithelial cell apoptosis, decreased expression of tight junction proteins, increased intestinal permeability and other barrier injuries occur in the chronic phase of human immunodeficiency virus (HIV) infection (7-12).

AIDS is one of the most widespread and destructive chronic infectious diseases to date (13-15). Persistent intestinal barrier damage, which leads to an heterotopia of microorganisms 
and induces a systemic inflammatory response, is the main factor that promotes the progression of chronic HIV infection to AIDS and leads to death (16). Therefore, it is important to perform research on the mechanisms underlying intestinal barrier damage to delay the progression of disease and prolong the life of patients. Currently, no effective strategy is available to inhibit the progression of gut barrier injury in patients with AIDS; thus, it is urgent to identify potential therapeutic targets.

MicroRNAs (miRNAs/miRs) are noncoding, single-stranded small RNA molecules of 22 nucleotides that are encoded by endogenous genes and are highly conserved in eukaryotic cells (17). miRNAs serve an important role in intestinal development, immune flora regulation, and intestinal barrier damage and repair. Yang et al (18) reported that miR-21 impaired intestinal barrier integrity by downregulating the Ras homolog gene family member B, and that miR-21 caused the disruption of tight junctions in intestinal epithelial cells, resulting in reduced transepithelial electrical resistance and increased mucosal permeability. Zhao et al ((19) reported that miR-124 directly targeted the aryl hydrocarbon receptor (AHR), which is inversely correlated within inflamed intestinal epithelial cells. AHR upregulation via the inhibition of miR-124 ameliorated experimental colitis (19). Another study demonstrated that inhibition of miR-146 attenuated lipopolysaccharide (LPS)-induced intestinal mucosal cell apoptosis (20). In addition, it was found that miR-125b-5p contributed to HIV-1 latency in resting primary CD4+ T lymphocytes (21). A previous miRNA sequencing study from our group showed that multiple miRNAs were abnormally expressed in the intestinal mucosa of HIV/AIDS patients, including miR-125b-5p (22). However, the functions and mechanisms of miR-125b-5p on intestinal mucosal cell injury remain unclear.

Intestinal barrier damage is the main factor that promotes the progression of AIDS. Glutamine transport in intestinal mucosa is mainly dependent on the sodium-dependent amino acid transport system, and alanine serine cysteine-preferring transporter 2 (ASCT2) is a main factor involved in this system (23). ASCT2 is one of the most important glutamine transporters in organisms and regulates the uptake of amino acids (24,25). Abnormal ASCT2 expression is associated with multiple tumors, including breast cancer (26), colorectal cancer (27), hepatocellular carcinoma (28), gastric cancer (29), and ovarian cancer (30). Our previous work has demonstrated that ASCT2 is abnormally low expressed in the damaged intestinal mucosa of AIDS patients, suggesting that ASCT2 may be associated with HIV-related intestinal mucosal barrier damage. However, the mechanism by which ASCT2 may regulate intestinal barrier injury and repair progression, especially HIV/AIDS progression, remains unknown.

The present study predicted that ASCT2 may be a candidate target of miR-125b-5p based on bioinformatics analysis. It was then hypothesized that miR-125b-5p might target ASCT2 to regulate intestinal barrier injury in patients with HIV/AIDS. First, miR-125b-5p and ASCT2 expression was determined in the intestinal mucosa of AIDS patients. Then, the potential role of miR-125b-5p on the regulation of ASCT2 was evaluated in an LPS-induced intestinal mucosa cell injury model in vitro. In addition, the role of miR-125b-5p in the PI3K/AKT/mTOR pathway was investigated, to further illuminate its regulatory mechanism.

\section{Materials and methods}

Clinical specimens and cell culture. Colon biopsy samples from male patients with AIDS and healthy subjects (both $n=10$; both aged 31-47 years) were collected from the First Affiliated Hospital of Kunming Medical University (Kunming, China) and used for RNA extraction. Patients and healthy subjects were enrolled between June and December 2014. Patients were diagnosed with AIDS according to the Guidelines for the Diagnosis and Treatment of AIDS (31). The exclusion criteria for patients were as follows: i) Patients with hepatitis $\mathrm{C}$, hepatitis B or syphilis; ii) patients with serious complications, such as malignant fat tumor or cardiovascular disease and iii) patients with systemic lupus erythematosus or rheumatoid arthritis. The exclusion criteria for healthy subjects were as follows: i) Chronic digestive disease and ii) abnormal routine physical and stool examination. Human intestinal embryonic mucosa tissue-derived cells (CCC-HIE-2) were purchased from the Cell Resource Center, Institute of Basic Medicine, Chinese Academy of Medical Sciences. CCC-HIE-2 cells were cultured in DMEM/High Glucose medium (cat. no. 11965092; Gibco; Thermo Fisher Scientific, Inc.) containing 10\% FBS (cat. no. S9020; Beijing Solarbio Science \& Technology Co., Ltd.) at $37^{\circ} \mathrm{C}$ and $5 \% \mathrm{CO}_{2}$. Cells were digested with $0.25 \%$ trypsin (cat. no. 25200-072; Gibco; Thermo Fisher Scientific, Inc.) for $30 \mathrm{sec}$ and washed with $1 \mathrm{X}$ PBS (cat. no. P1020; Beijing Solarbio Science \& Technology Co., Ltd.). Then, $2 \times 10^{4}$ cells were plated on a $10-\mathrm{cm}^{2}$ cell dish with $10 \mathrm{ml}$ medium and cultured until the cells were in a good proliferation state.

LPS treatment of CCC-HIE-2 cells. CCC-HIE-2 cells $\left(\sim 5 \times 10^{5}\right)$ were plated on a $10-\mathrm{cm}^{2}$ cell dish with regular DMEM medium prior to treatment. The next day, cells at $70 \%$ confluency were treated with DMEM medium containing 20, 40, 80, 100 and $150 \mathrm{ng} / \mathrm{ml}$ LPS (Sigma-Aldrich; Merck KGaA) to induce apoptosis. After $48 \mathrm{~h}$, the cells were collected, and untreated CCC-HIE-2 cells were used as a control group. MTT assay and flow cytometry were performed to investigate cell damage. Ultimately, $100 \mathrm{ng} / \mathrm{ml}$ was selected as the optimum LPS concentration in subsequent experiments.

MTT assay. Induced or transfected cells were digested with $0.25 \%$ trypsin to create a single-cell suspension. Cells were counted, and $2.5 \times 10^{4}$ cells were inoculated onto a 96 -well plate for $24 \mathrm{~h}$. The culture solution was aspirated with a lance and carefully washed twice with PBS. A total of $100 \mu \mathrm{l}$ of fresh culture solution with a final concentration of $0.5 \mathrm{mg} / \mathrm{ml}$ MTT (cat. no. M1020; Beijing Solarbio Science \& Technology Co., Ltd.) were added to each well and cultured for $4 \mathrm{~h}$. After the MTT solution was discarded and $100 \mu \mathrm{l}$ DMSO was added to each well, the culture plate was placed on an oscillator and shaken for $10 \mathrm{~min}$ to fully dissolve the purple crystals. The absorbance values of each well were measured at $570 \mathrm{~nm}$ using a microplate reader (Bio-Rad Laboratories, Inc.), and the cell survival rate was calculated.

Flow cytometry. An Annexin V-FITC Apoptosis Detection kit purchased from Beyotime Institute of Biotechnology was used to analyze cell apoptosis by flow cytometry. Briefly, $1 \times 10^{6}$ cells were inoculated onto a 6-well plate and cultured for $24 \mathrm{~h}$. Each 
sample was prepared in triplicate. Following treatment with LPS for $48 \mathrm{~h}$, and transfections with ASCT2-overexpressing plasmid and/or miR-125b-5p mimic/inhibitor for $24 \mathrm{~h}$, the cells were harvested via $0.25 \%$ trypsin digestion, incubated with $10 \mu \mathrm{l}$ Annexin V-FITC at $4^{\circ} \mathrm{C}$ for $30 \mathrm{~min}$ and then incubated with $5 \mu$ l propidium iodide (PI) solution for $15 \mathrm{~min}$ in the dark. The stained cells were detected using BD FACSCanto II flow cytometry (BD Biosciences) with CellQuest software (BD Biosciences). The early apoptotic cells were Annexin V-FITC-positive and PI-negative, and late apoptotic cells were Annexin V-FITC-positive and PI-positive. The apoptotic rate of cells is presented as the percentage of cells in early and late apoptotic phases.

Cell transfection. CCC-HIE-2 cells were digested with $0.25 \%$ trypsin, and cell suspensions (200 cells/ $\mu 1)$ were prepared $\left(5 \times 10^{6}\right.$ cells in a 6 -well plate). When the cells reached $70 \%$ confluency, they were treated with LPS for $48 \mathrm{~h}$, and then transfected with different concentrations of miR-125b-5p mimic or inhibitor (cat. nos. MC10148 and MH10148, respectively; Thermo Fisher Scientific, Inc.), or $4 \mu \mathrm{g}$ ASCT2/pEGFP plasmid with $6 \mu$ l Lipofectamine 2000 (Invitrogen; Thermo Fisher Scientific, Inc.) for $24 \mathrm{~h}$, according to the instructions of the manufacturer. After $6 \mathrm{~h}$, the transfection media were replaced with fresh DMEM/High glucose (cat. no. 11965092; Gibco; Thermo Fisher Scientific, Inc.) containing $10 \%$ fetal bovine serum (cat. no. S9020; Beijing Solarbio Science \& Technology Co., Ltd.). miRNA mimics and inhibitors are single-chain oligonucleotides that are used to investigate gain-of-function or loss-of-function effects, respectively. ASCT2 gene was inserted into pEGFP plasmid with restriction enzyme cutting site $K p n \mathrm{I}$ and XhoI. ASCT2/pEGFP plasmid was sequenced by Shanghai Sangon Biotech Co., Ltd. The sequences of miR-125b-5p mimic and inhibitor are as follows: hsa-miR-125b-5p mimic, 5'-UCCCUGAGACCCUAACUUGUCA-3'; has-miR-125b-5p inhibitor, UCACAAGUUAGGGUCUCAGGGA-3'; mimic negative control (NC), 5'-UUCUCCGACGUGUCACGUTT-3'; and inhibitor NC, 5'-CAGUACUUUUGUGUAGUACAA-3'.

Reverse transcription-quantitative PCR (RT-qPCR). Total RNA was extracted from samples using TRIzol reagent (cat. no. 15596018; Invitrogen; Thermo Fisher Scientific, Inc.), and miRNA was extracted using the miRNeasy Mini kit (cat. no. 217004; Qiagen $\mathrm{GmbH}$ ). After quantifying the concentration and purity of RNA with a DyNA Quant 200 nucleic acid concentration tester (GE Healthcare), the RNA was reverse transcribed into cDNA according to the instructions of the PrimeScript RT Reagent kit (cat. no. RR036Q; Takara Biotechnology Co., Ltd.), and miRNA was reverse transcribed using the miRNA First-Strand Synthesis kit (cat. no. 638313; Clontech Laboratories, Inc.) according to the manufacturer's instructions. RT-qPCR was performed using TB Green Premix Ex Taq (cat. no. RR420A; Takara Biotechnology Co., Ltd.) or miRNA qRT-PCR TB Green kit (cat. no. 638314; Clontech Laboratories, Inc.) on a 7500 Real-time PCR system (Applied Biosystems; Thermo Fisher Scientific, Inc.). RT-qPCR was performed as follows: Initial denaturation, $95^{\circ} \mathrm{C}$ for $10 \mathrm{~min}$, followed by 40 cycles of $95^{\circ} \mathrm{C}$ for $15 \mathrm{sec}$ and $60^{\circ} \mathrm{C}$ for $60 \mathrm{sec}$. The results were analyzed using the $2^{-\Delta \Delta \mathrm{Cq}}$ method (32). $\beta$-actin or U6 served as the internal controls for the mRNA or miRNA qPCR assays, respectively. The following primers were used in the present study: ASCT2, forward 5'-TGTGTAGAGGAGAA GAATGG-3' and reverse 5'-GATGGCAAGAGTGAGGAC-3'; $\beta$-actin, forward 5'-GGCCTCCAAGGAGTAAGACC-3' and reverse-5'-AGGGGTCTACATGGCAACTG-3'; miR-125b-5p, forward 5'-TCCCTGAGACCCTAACTTGTGA-3' and reverse 5'-AGTCTCAGGGTCCGAGGTATTC-3'; and U6, forward 5'-CTCGCTTCGGCAGCACA-3' and reverse 5'-AACGCTTC ACGAATTTGCGT-3'.

Western blot analysis. The cell culture medium was discarded. Cells were washed with PBS, collected and lysed using RIPA lysis buffer (cat. no. P0013B; Beyotime Institute of Biotechnology) for protein extraction. The protein concentration was quantified using a BCA kit (cat. no. P0012; Beyotime Institute of Biotechnology). Equal amounts $(30 \mu \mathrm{g})$ of protein lysates were separated by $12 \%$ SDS-PAGE (cat. no. P0012A; Beyotime Institute of Biotechnology) and then transferred onto PVDF membranes (cat. no. FFP36; Beyotime Institute of Biotechnology). The membranes were blocked with 5\% nonfat milk (cat. no. P0216; Beyotime Institute of Biotechnology) for $2 \mathrm{~h}$ at $4{ }^{\circ} \mathrm{C}$ and then incubated with primary antibodies against ASCT2 (1:1,000; cat. no. ab237704; Abcam), GAPDH (1:2,000; cat. no. ab9485; Abcam), phosphorylated (p-) AKT (1:1,000; cat. no. ab81283; Abcam), AKT (1:1,000; cat. no. ab8805; Abcam), p-PI3K (1:1,000; cat. no. ab191606; Abcam), PI3K (1:1,000; cat. no. ab40776; Abcam), p-mTOR (1:1,000; cat. no. ab109268; Abcam) and mTOR (1:1,000; cat. no. ab134903; Abcam) overnight at $4^{\circ} \mathrm{C}$. After washing with $1 \mathrm{X}$ PBST (cat. no. P1031; Beijing Solarbio Science \& Technology Co., Ltd.), the membranes were probed with HRP-conjugated goat anti-rabbit IgG secondary antibodies (1:5,000; cat. no. ab7090; Abcam) for $2 \mathrm{~h}$ at room temperature. Then, protein bands were observed using an ECL chemiluminescence kit (cat. no. P0018S; Beyotime Institute of Biotechnology) on the Bio-best type 140E Gel Imaging Analysis system (SIM International Group Co., Ltd.). GAPDH served as an internal control. ImageJ 2.x software was used to quantify the protein bands (National Institutes of Health).

Dual luciferase reporter assay. The relationship between miR-125b-5pandASCT2 was examined using aDual-Luciferase Reporter Assay kit (cat. no. E1910; Promega Corporation). A wild-type (WT) or mutant (MUT) version of the binding site in the 3'-untranslated region (3'-UTR) of ASCT2 was cloned into the pmiRGLO vector (Promega Corporation). Cells were co-transfected with the miR NC or miR-125b-5p mimic and with the pmiRGLO-ASCT2-WT or pmiRGLO-ASCT2-MUT reporter plasmids by Lipofectamine 2000 (cat. no. 11668-027; Invitrogen; Thermo Fisher Scientific, Inc.). After $48 \mathrm{~h}$ of culture, the luciferase reporter system was used to analyze the luciferase activity. The relative luciferase activity of CCC-HIE-2 cells was expressed as the ratio of firefly luciferase activity relative to sea kidney luciferase activity.

Statistical analysis. Data are expressed as the mean \pm standard deviation. Differences between two groups were compared using the Student's t-test. Differences among three or more groups were compared using one-way ANOVA with Tukey's 
A

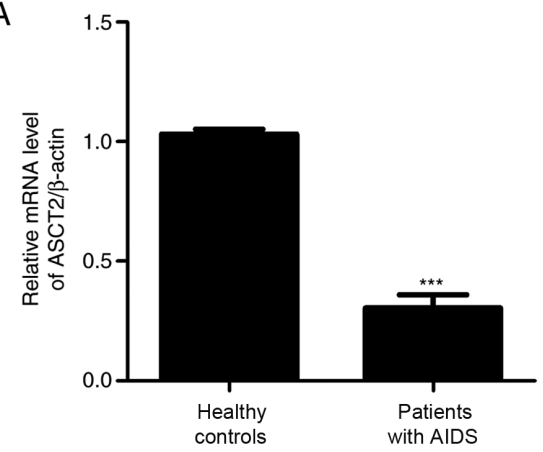

B

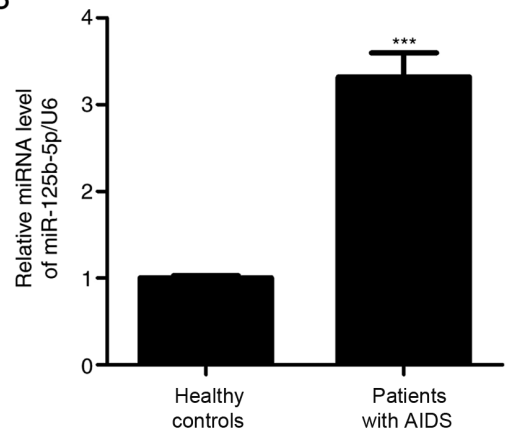

Figure 1. miR-125b-5p and ASCT2 expression levels in patients with AIDS. Colon biopsy samples from patients with AIDS (n=10) and from healthy control subjects $(\mathrm{n}=10)$ were collected, and RT-qPCR was performed to detect (A) miR-125b-5p expression levels and (B) ASCT2 mRNA expression levels. All experiments were repeated at least thrice. Data are expressed as the mean $\pm \mathrm{SD} .{ }^{* * *} \mathrm{P}<0.001$ compared with healthy controls. miR, microRNA; ASCT2, alanine serine cysteine-preferring transporter 2 .

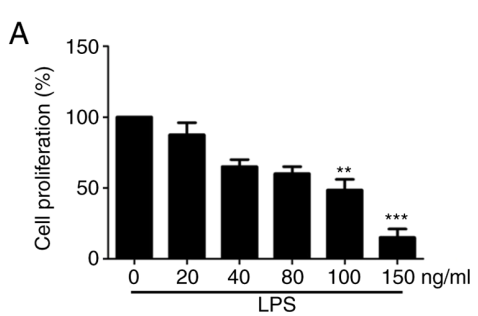

B

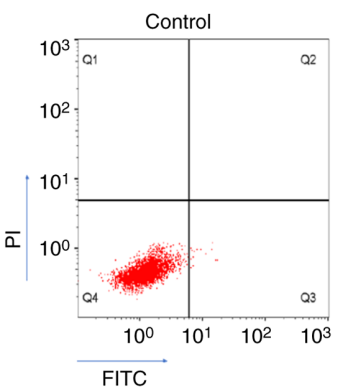

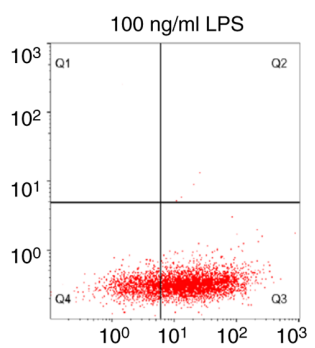

E
C

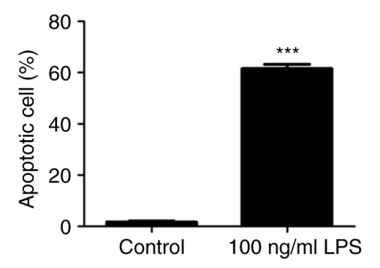

D

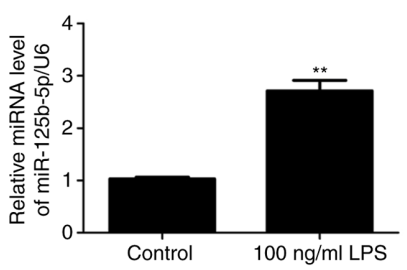

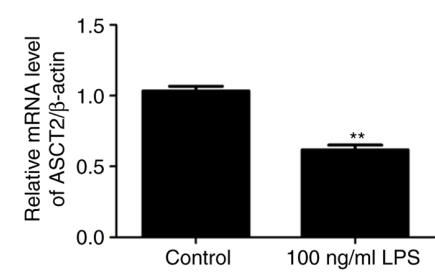

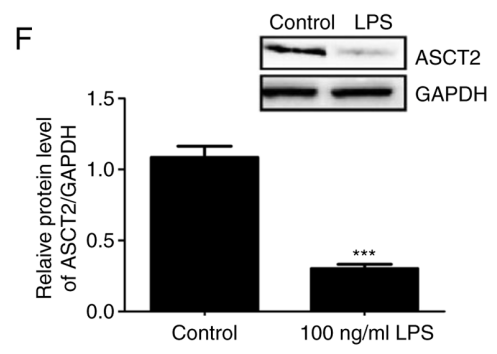

Figure 2. miR-125b-5p and ASCT2 expression levels in the LPS-induced intestinal mucosa cell injury model. (A) Cell viability was assessed after CCC-HIE-2 cells were treated with different concentrations of LPS for $48 \mathrm{~h}$. (B) Representative flow cytometry plots and (C) quantification of apoptosis in CCC-HIE-2 cells treated with $100 \mathrm{ng} / \mathrm{ml}$ LPS for $48 \mathrm{~h}$. (D) miR-125b-5p expression levels and (E) ASCT2 mRNA expression levels in CCC-HIE-2 cells treated with $100 \mathrm{ng} / \mathrm{ml}$ LPS for $48 \mathrm{~h}$. (F) ASCT2 protein expression was measured by western blot analysis. Data are expressed as the mean \pm SD $(\mathrm{n}=5)$. ${ }^{* *} \mathrm{P}<0.01$ and ${ }^{* * * *} \mathrm{P}<0.001$ compared with the control group ( $0 \mathrm{ng} / \mathrm{ml} \mathrm{LPS})$. miR, microRNA; ASCT2, alanine serine cysteine-preferring transporter 2; LPS, lipopolysaccharide.

post hoc test. Statistical analyses were performed with GraphPad Prism 5 software (GraphPad Software Inc.). $\mathrm{P}<0.05$ was considered to indicate a statistically significant difference.

\section{Results}

miR-125b-5p and ASCT2 expression levels in the intestinal mucosa of patients with AIDS. To measure miR-125b-5p and ASCT2 expression levels, colon biopsy samples collected from 10 AIDS patients were examined by RT-qPCR. As shown in Fig. 1, miR-125b-5p expression levels were significantly increased, whereas ASCT2 expression levels were significantly decreased in the AIDS group compared with the control healthy group. These results suggested that miR-125b-5p and ASCT2 may regulate intestinal barrier injury in patients with AIDS.

miR-125b-5p and ASCT2 expression levels in the LPS-induced intestinal mucosa cell injury model. An in vitro intestinal mucosal cell injury model was constructed using CCC-HIE-2 
A

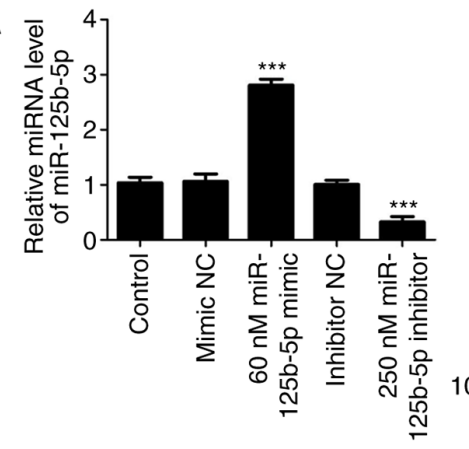

B

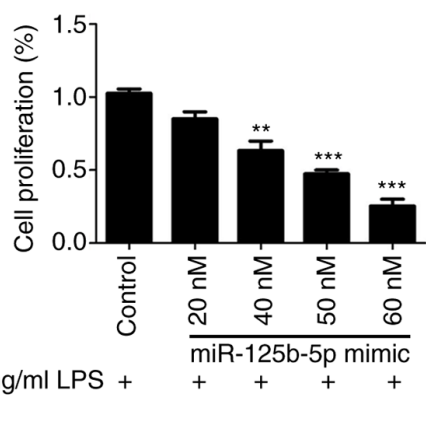

C

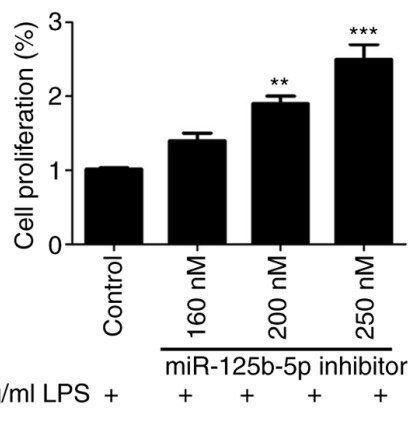

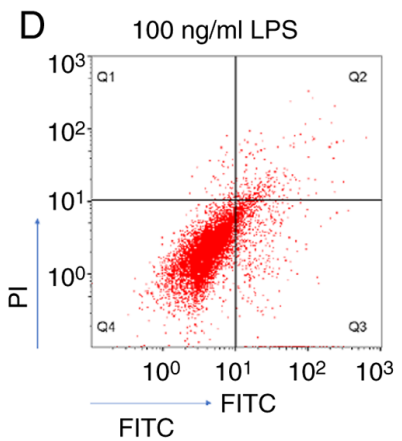
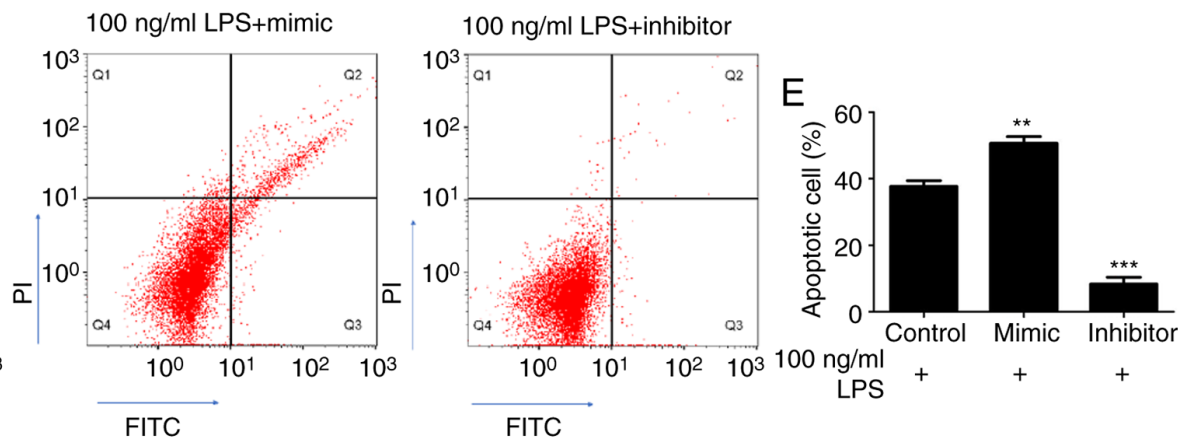

Figure 3. Effect of miR-125b-5p on LPS-induced intestinal mucosa cell injury. CCC-HIE-2 cells were transfected with the miR-125b-5p mimic or inhibitor for $24 \mathrm{~h}$ after treatment with LPS for $48 \mathrm{~h}$. (A) The transfection efficiency of miR-125b-5p mimic and inhibitor was verified by RT-qPCR assay. (B and C) Cell viability assay. (D) Representative flow cytometry plots and (E) quantification of apoptosis. Data are expressed as the mean $\pm \mathrm{SD}(\mathrm{n}=5) .{ }^{* *} \mathrm{P}<0.01$ and ${ }^{* * * *} \mathrm{P}<0.001$ vs. $100 \mathrm{ng} / \mathrm{ml}$ LPS-alone. miR, microRNA; LPS, lipopolysaccharide; NC, negative control.

cells treated with different concentrations of LPS. Cell proliferation was then measured using the MTT assay. The results demonstrated that $100 \mathrm{ng} / \mathrm{ml}$ and $150 \mathrm{ng} / \mathrm{ml}$ LPS significantly inhibited CCC-HIE-2 cell proliferation compared with $0 \mathrm{ng} / \mathrm{ml}$ LPS (Fig. 2A). Therefore, the $100 \mathrm{ng} / \mathrm{ml}$ LPS dose was used in subsequent experiments. Apoptosis of the injured cells was detected by flow cytometry. The results showed that cell apoptosis rates significantly increased following treatment with LPS (100 ng/ml) (Fig. 2B and C), suggesting that the in vitro intestinal mucosa cell injury model was successfully established. The expression levels of miR-125b-5p and ASCT2 in the LPS-injured CCC-HIE-2 cells were detected by RT-qPCR and western blotting. The RT-qPCR results revealed that miR-125b-5p expression levels were significantly increased (Fig. 2D), whereas ASCT2 expression levels were decreased (Fig. 2E) in LPS-injured CCC-HIE-2 cells compared with control cells. Western blotting results showed that the differences in ASCT2 protein levels were consistent with the observed differences in mRNA levels (Fig. 2F). These results implied that the in vitro intestinal mucosa cell injury model was successfully established using LPS-induced CCC-HIE-2 cells.

Effect of miR-125b-5p on proliferation and apoptosis of the $L P S$-induced intestinal mucosa cell injury model. First, the transfection efficiency of miR-125b-5p mimic and inhibitor was confirmed in CCC-HIE-2 cells. As shown in the Fig. 3A, compared with their respective control groups, the expression levels of miR-125b-5p were significantly increased in the $60 \mathrm{nM}$ miR-125b-5p mimic group, and significantly decreased in the $250 \mathrm{nM}$ miR-125b-5p inhibitor group. There were no significant differences between the mimic NC group, the inhibitor NC group and the untransfected cell control group (Fig. 3A). Subsequently, to investigate the effect of miR-125b-5p levels on LPS-induced CCC-HIE-2 cell injury, LPS-treated CCC-HIE-2 cells were transfected with the miR-125b-5p mimic or inhibitor, and MTT assays and flow cytometry were performed to examine cell proliferation and apoptosis. MTT assay results showed that the miR-125b-5p mimic significantly inhibited LPS-treated CCC-HIE-2 cell proliferation in a concentration-dependent manner (Fig. 3B). By contrast, the miR-125b-5p inhibitor significantly increased LPS-treated CCC-HIE-2 cell proliferation in a concentration-dependent manner (Fig. 3C). Therefore, the doses of $60 \mathrm{nM}$ miR-125b-5p mimic and $250 \mathrm{nM}$ inhibitor were selected to perform the subsequent experiments. As shown in Fig. 3D and E, transfection with the miR-125b-5p mimic increased cell apoptosis, while transfection with the miR-125b-5p inhibitor inhibited apoptosis, in the LPS-treated CCC-HIE-2 cell model.

ASCT2 is negatively regulated by miR-125b-5p. To explore the mechanism of miR-125b-5p in LPS-induced intestinal mucosa cell injury, the potential targets of miR-125b-5p were investigated using TargetScan (33). The TargetScan analysis predicted that ASCT2 contains a sequence that is potentially complementary to miR-125b-5p (Fig. 4A). To validate this prediction, WT and MUT luciferase reporter vectors targeting the 3'-UTR of ASCT2 were constructed and transfected into LPS-treated CCC-HIE-2 cells. Dual luciferase reporter assays were conducted to examine the relationship between ASCT2 and miR-125b-5p. As shown in Fig. 4B, luciferase activity was reduced by the miR-125b-5p mimic in cells transfected with the ASCT2-WT reporter, whereas it was unchanged in 

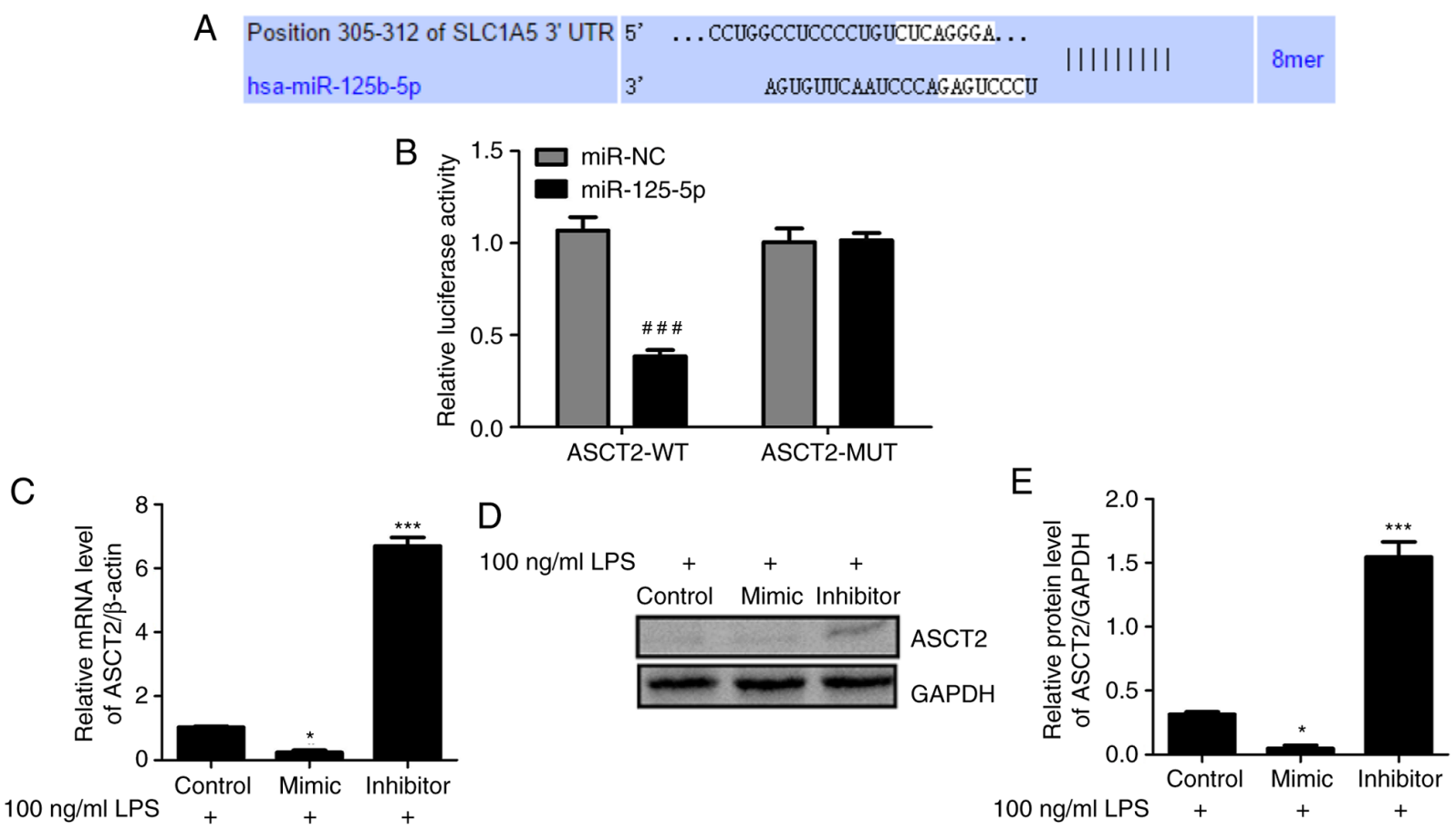

Figure 4. ASCT2 is a direct target of miR-125b-5p. (A) The potential binding sites of miR-125b-5p and ASCT2 were predicted by TargetScan. (B) A luciferase reporter assay was performed in CCC-HIE-2 cells to confirm the interaction between miR-125b-5p and ASCT2. (C) Effects of miR-125b-5p mimic or inhibitor on ASCT2 mRNA expression levels. (D) Representative blot images and (E) quantification of ASCT2 protein expression levels following transfection with miR-125b-5p mimic or inhibitor. Data are expressed as the mean $\pm \mathrm{SD}(\mathrm{n}=5)$. ${ }^{\# \# *} \mathrm{P}<0.001$ compared with miR-NC; ${ }^{*} \mathrm{P}<0.05$ and ${ }^{* * * *} \mathrm{P}<0.001$ vs. LPS-alone. ASCT2, alanine serine cysteine-preferring transporter 2; miR, microRNA; NC, negative control; UTR, untranslated region; WT, wild-type; MUT, mutant; LPS, lipopolysaccharide.
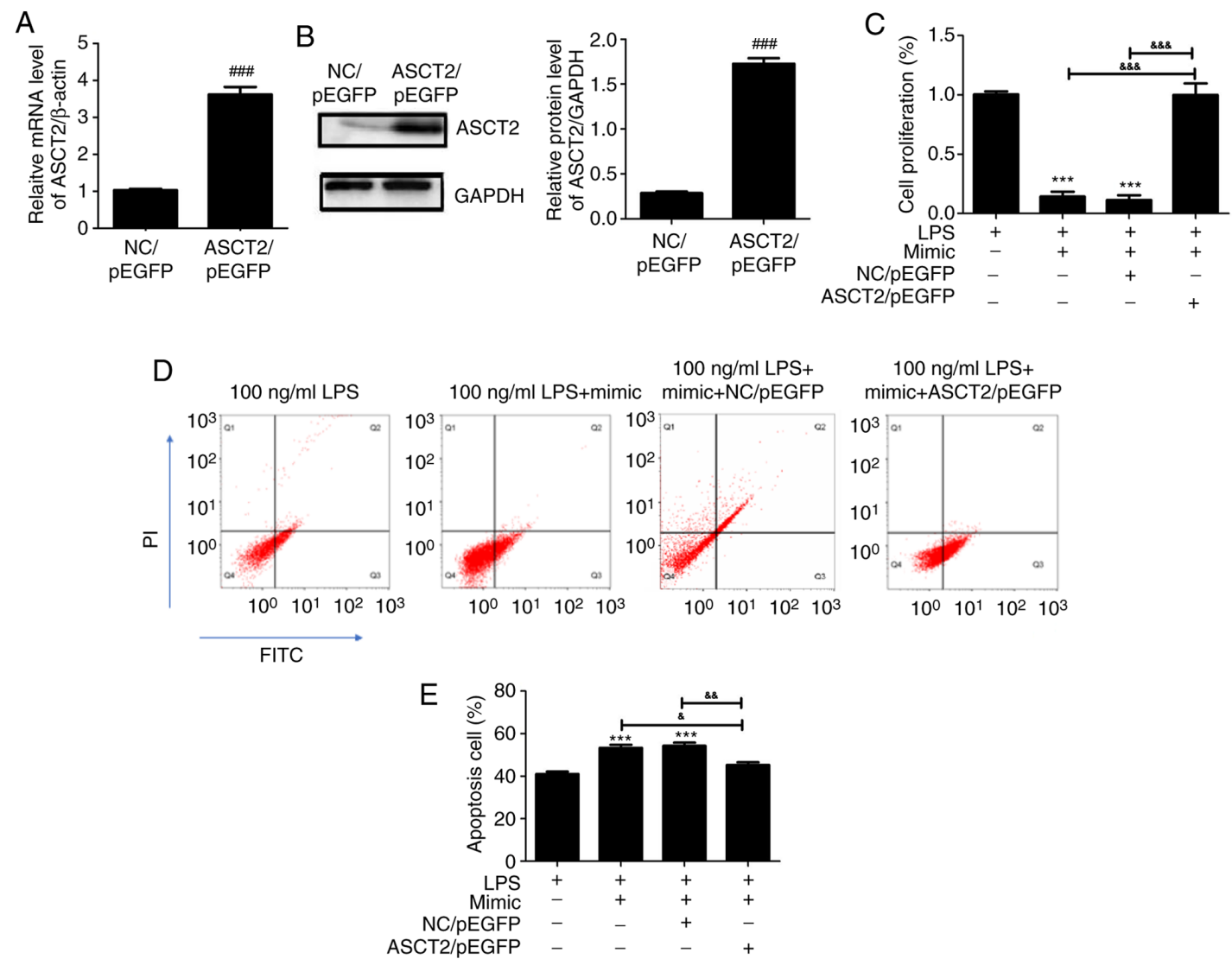

Figure 5. Rescue effect of ASCT2 overexpression on miR-125b-5p-mediated regulation of LPS-induced intestinal mucosa cell injury. (A and B) ASCT2 is successfully overexpressed following transfection with the ASCT2/pEGFP plasmid for $48 \mathrm{~h}$. An empty vector plasmid was used as a control. (C) Cell viability assay. (D) Representative flow cytometry plots and (E) quantification of apoptosis. Data are expressed as the mean \pm SD and were analyzed ( $\mathrm{n}=5$ ). ${ }^{\# \# /} \mathrm{P}<0.001$ compared with NC/pEGFP; ${ }^{* * *} \mathrm{P}<0.001$ vs. LPS-alone; ${ }^{\text {}} \mathrm{P}<0.05,{ }^{\text {\&\&}} \mathrm{P}<0.01,{ }^{\& \&} \& \mathrm{P}<0.001$. 

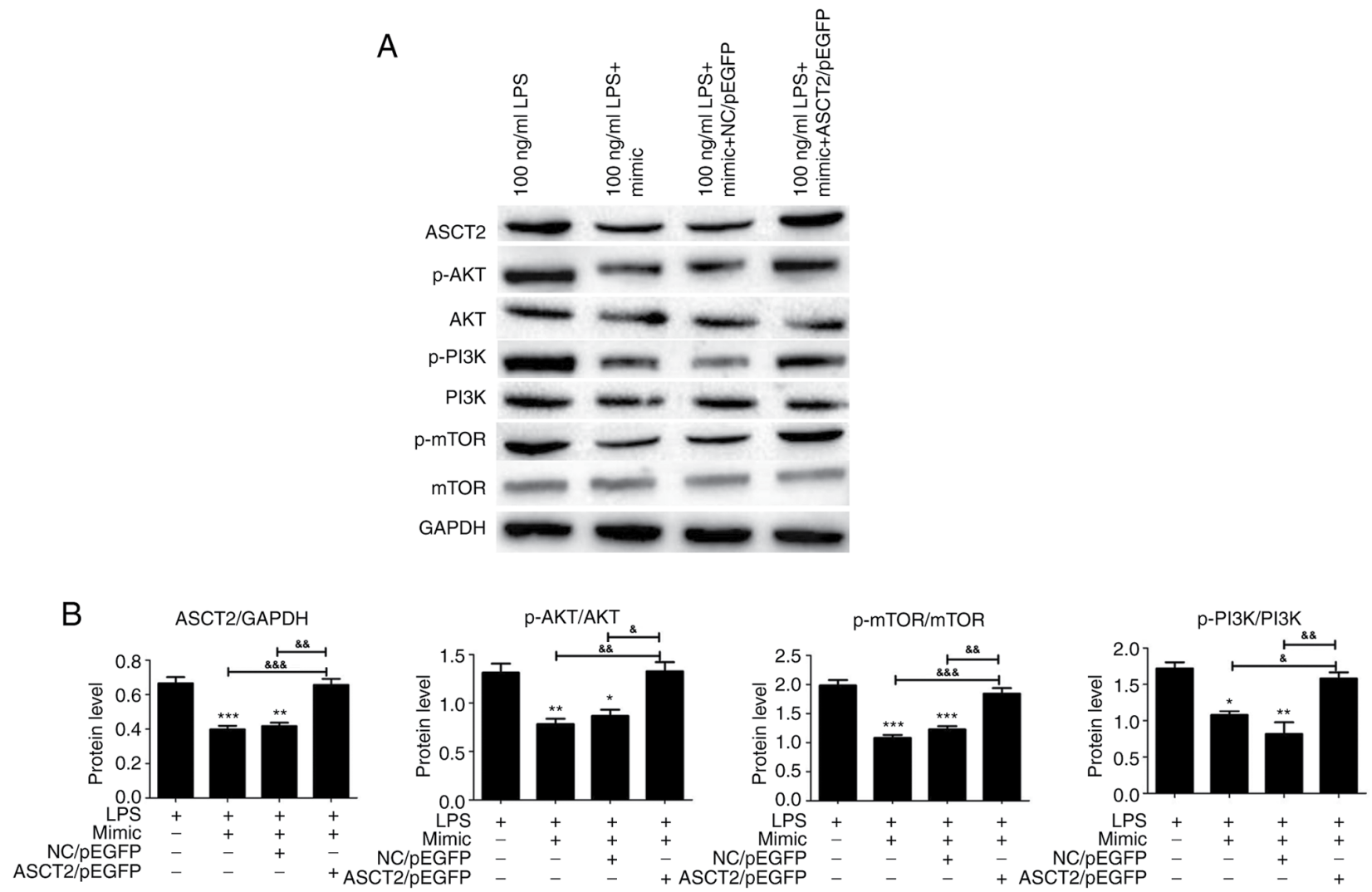

Figure 6. PI3K/AKT/mTOR pathway expression in the miR-125b-5p and ASCT2-transfected LPS-induced intestinal mucosa cell injury model. CCC-HIE-2 cells were transfected with the miR-125b-5p mimic alone, the miR-125b-5p mimic + NC/pEGFP, or the miR-125b-5p mimic + ASCT2/pEGFP for $24 \mathrm{~h}$ following $48 \mathrm{~h}$ treatment with LPS, and the expression levels of proteins involved in the PI3K/AKT/mTOR pathway were detected by western blot analysis. (A) Representative blot images. (B) Quantitative western blot results. Data are expressed as the mean $\pm \mathrm{SD}(\mathrm{n}=5)$. ${ }^{*} \mathrm{P}<0.05,{ }^{* *} \mathrm{P}<0.01,{ }^{* * * *} \mathrm{P}<0.001$ vs. LPS-alone; ${ }^{\&} \mathrm{P}<0.05,{ }^{\& \&} \mathrm{P}<0.01,{ }^{\& \& \&} \mathrm{P}<0.001$. miR, microRNA; ASCT2, alanine serine cysteine-preferring transporter 2; LPS, lipopolysaccharide; NC, negative control; $\mathrm{p}-$, phosphorylated.

cells transfected with the ASCT2-MUT reporter. In addition, RT-qPCR and western blot data indicated that the miR-125b-5p mimic inhibited ASCT2 mRNA and protein expression levels, whereas the miR-125b-5p inhibitor promoted ASCT2 mRNA and protein expression levels in LPS-treated CCC-HIE-2 cells (Fig. 4C-E). These results revealed that miR-125b-5p negatively regulated ASCT2 expression in the LPS-induced intestinal mucosa cell injury model.

miR-125b-5p inhibits cell proliferation and induces apoptosis in LPS-treated CCC-HIE-2 cells by regulating ASCT2. To clarify whether the upregulation of ASCT2 could rescue the miR-125b-5p-mediated effects, an ASCT2/pEGFP plasmid was transfected into LPS-treated CCC-HIE-2 cells. Compared with the empty vector control (NC/pEGFP), the mRNA (Fig. 5A) and protein (Fig. 5B) expression levels of ASCT2 were significantly increased following transfection with the ASCT2/pEGFP plasmid. As presented in Fig. 5C, the miR-125b-5p mimic inhibited LPS-treated CCC-HIE-2 cell proliferation, and this effect was reversed by co-transfection with the miR-125b-5p mimic and the ASCT2/pEGFP plasmid (Fig. 5C). Furthermore, cell apoptosis was promoted by the miR-125b-5p mimic in LPS-treated CCC-HIE-2 cells, but this was partially rescued by co-transfection with the miR-125b-5p mimic and the ASCT2/pEGFP plasmid (Fig. 5D and E). These findings demonstrated that ASCT2 overexpression could alleviate LPS-induced intestinal mucosal cell injury. The present study demonstrated a negative correlation between ASCT2 and miR-125b-5p expression in LPS-induced intestinal mucosa cell injury; of note, ASCT2 was involved in the miR-125b-5p-mediated inhibition of LPS-treated CCC-HIE-2 cell progression.

miR-125b-5p inhibits the PI3K/AKT/mTOR signaling pathway in the LPS-induced intestinal mucosa cell injury model. Previous studies have demonstrated that the mTOR pathway has a mechanistic role in the development of intestinal epithelial injury and repair $(34,35)$. Therefore, the present study investigated the expression levels of PI3K/AKT/mTOR pathway-related proteins to further explore the possible effects of miR-125b-5p on the LPS-induced intestinal mucosa cell injury model. PI3K, p-PI3K, AKT, p-AKT, mTOR and p-mTOR protein expression levels in LPS-treated CCC-HIE-2 cells were detected by western blot. analysis Transfection with the miR-125b-5p mimic significantly decreased PI3K, AKT and mTOR phosphorylation levels (Fig. 6). Notably, ASCT2 overexpression partially reversed this effect (Fig. 6). Therefore, miR-125b-5p inhibited the PI3K/AKT/mTOR signaling pathway in the LPS-induced intestinal mucosa cell injury model. This finding suggested that miR-125b-5p may regulate LPS-induced intestinal mucosa cell injury through the PI3K/AKT/mTOR signaling pathway. 


\section{Discussion}

Serious injury to the intestinal barrier leads to microbial heterotopia, which is common in patients with HIV/AIDS. Microbial products, such as LPS and bacterial 16S RNA, have been detected in the blood of AIDS patients since the early days of the epidemic (36-38). Blood LPS concentrations were detected in AIDS patients and healthy controls in the present study and the results revealed that the blood LPS concentration of AIDS patients was higher compared with that of healthy controls (data not shown). Thus, an in vitro intestinal mucosal cell injury model was established for the present study with LPS treatment.

miRNAs are major regulators of cell function and homeostasis (39). miRNAs are involved in many physiological and pathological processes, including cell differentiation, proliferation, signal transduction and apoptosis, and their abnormal expression is closely related to the occurrence and development of many diseases (40). miR-122a is implicated in the regulation of intestinal epithelial tight junctions during inflammation, and TNF- $\alpha$ induces the epithelial expression of miR-122a, thereby causing the degradation of its target gene occludin and resulting in increased mucosal permeability (41). miRNAs serve an important role in the replication of HIV-infected viruses (42). The JunD gene inhibits proliferation of intestinal epithelial cells by increasing miR-29b levels transcriptionally and post-transcriptionally (43). Because miRNAs are highly conserved and have good stability in the extracellular domain, miRNAs could serve as markers for disease diagnosis. With the deepening of the study on the related role of miRNAs in human physiological diseases, the potential of miRNAs as a drug target has emerged and become a research hotspot. However, studies on miRNAs in the repair of intestinal barrier injury in HIV/AIDS patients are rarely reported.

The present study found that ASCT2 expression in colon samples from patients with HIV/AIDS was downregulated and negatively correlated with miR-125b-5p expression. Huang et al (21) reported that miR-125b-5p targets the 3 long terminal repeat region of the HIV genome, leading to differential HIV-1 infectivity/resistance to different cells in various developmental stages. The present study established an LPS-induced CCC-HIE-2 cell injury model and examined the effects of miR-125b-5p and ASCT2 on LPS-induced intestinal mucosal injury in vitro. Dual luciferase reporter assays were used to identify ASCT2 as a direct target of miR-125b-5p in intestinal mucosa injury cells, suggesting that the suppression of ASCT2 by miR-125b-5p may be a novel therapeutic approach for intestinal mucosa injury in patients with HIV/AIDS.

In addition, the current results confirmed that the effect of miR-125b-5p and ASCT2 on intestinal mucosa injury may be related to the $\mathrm{PI} 3 \mathrm{~K} / \mathrm{AKT} / \mathrm{mTOR}$ pathway. A previous study indicated that elevated ASCT2 expression promoted lung cell growth and survival through mTOR signaling (44). Activation of the PI3K/AKT signaling pathway inhibits cell apoptosis and facilitates the protective effect of magnesium sulfate against intestinal ischemia-reperfusion injury (45). Therefore, the present study focused on the PI3K/AKT/mTOR signaling pathway when assessing the effects of miR-125b-5p and ASCT2 in the intestinal mucosa injury model. The results indicated that miR-125b-5p upregulation decreased the expression levels of p-AKT, p-PI3K, and p-mTOR in LPS-induced intestinal mucosa cells, whereas ASCT2 overexpression promoted p-AKT, p-PI3K, and p-mTOR expression. The PI3K/AKT/mTOR pathway is involved in intestinal epithelial barrier dysfunction following severe burn (46). The levels of p-PI3K, p-AKT, and p-mTOR were decreased under miR-125b-5p treatment and increased following ASCT2 overexpression, indicating that miR-125b-5p downregulated ASCT2 expression by targeting the 3'-UTR of ASCT2 and then promoted the injury of LPS-induced intestinal mucosa cells through the PI3K/AKT/mTOR pathway.

In conclusion, the present study demonstrated that miR-125b-5p overexpression inhibited growth in the LPS-induced intestinal mucosal cell model by targeting ASCT2 expression and inhibiting the PI3K/AKT/mTOR pathway. By contrast, the inhibition of miR-125b-5p alleviated LPS-induced intestinal mucosal cell injury by increasing ASCT2 expression and promoting the PI3K/AKT/mTOR pathway. The present study was the first to investigate the relationship between miR-125b-5p and ASCT2 in LPS-induced intestinal mucosa cells and provided new insight into the molecular mechanisms of intestinal barrier injury in HIV/AIDS progression. These findings may indicate a path towards a novel treatment for intestinal barrier injury in patients with HIV/AIDS.

\section{Acknowledgements}

Not applicable.

\section{Funding}

This work was supported by the National Scientific Foundation of China (grant no. 81870458), the Special Project of Yunnan Science and Technology Department-Kunming Medical University Applied Basic Research [grant no. 2019FE001(-196)], the Yunnan Engineering Technology Center of Digestive Disease (grant no. 2018DH006), and the Project for Innovation Team of Yunnan Provincial Science and Technology Department (grant no. 2018HC005).

\section{Availability of data and materials}

The datasets used and/or analyzed during the current study are available from the corresponding author on reasonable request.

\section{Authors' contributions}

HG and KW conceived and designed the study. JG, YQ, HW and JL performed the experiments. HG, QP and $\mathrm{YZ}$ processed data. $\mathrm{HG}, \mathrm{JG}$ and $\mathrm{KW}$ wrote the manuscript. $\mathrm{HG}$ and $\mathrm{KW}$ reviewed and edited the manuscript. $\mathrm{HG}$ and $\mathrm{KW}$ confirm the authenticity of all the raw data. All authors read and approved the manuscript.

\section{Ethics approval and consent to participate}

All human tissue samples were collected at the First Affiliated Hospital of Kunming Medical University, and written 
informed consent was obtained from all patients. All methods were approved by the Research Medical Ethics Committee of Kunming Medical University (Kunming, China) and were performed in accordance with the approved guidelines.

\section{Patient consent for publication}

Not applicable.

\section{Competing interests}

The authors declare that they have no competing interests.

\section{References}

1. Druml W: Intestinaler Crosstalk. Med Klin Intensivmed Notfmed 113: 470-477, 2018 (In German).

2. Groschwitz KR and Hogan SP: Intestinal barrier function: Molecular regulation and disease pathogenesis. J Allergy Clin Immunol 124: 3-20, quiz 21-22, 2009.

3. Hermiston ML and Gordon JI: Inflammatory bowel disease and adenomas in mice expressing a dominant negative $\mathrm{N}$-cadherin. Science 270: 1203-1207, 1995.

4. Costantini TW, Peterson CY, Kroll L, Loomis WH, Eliceiri BP, Baird A, Bansal V and Coimbra R: Role of p38 MAPK in burn-induced intestinal barrier breakdown. J Surg Res 156: 64-69, 2009.

5. Yasuda T, Takeyama Y, Ueda T, Shinzeki M, Sawa H, Nakajima T and Kuroda Y: Breakdown of intestinal mucosa via accelerated apoptosis increases intestinal permeability in experimental severe acute pancreatitis. J Surg Res 135: 18-26, 2006.

6. Sabatino A, Regolisti G, Cosola C, Gesualdo L and Fiaccadori E: Intestinal microbiota in type 2 diabetes and chronic kidney disease. Curr Diab Rep 17: 16, 2017.

7. Vyboh K, Jenabian MA, Mehraj V and Routy JP: HIV and the gut microbiota, partners in crime: Breaking the vicious cycle to unearth new therapeutic targets. J Immunol Res 2015: 614127, 2015.

8. Nazli A, Chan O, Dobson-Belaire WN, Ouellet M, Tremblay MJ, Gray-Owen SD, Arsenault AL and Kaushic C: Exposure to HIV-1 directly impairs mucosal epithelial barrier integrity allowing microbial translocation. PLoS Pathog 6: e1000852, 2010.

9. Kristoff J, Haret-Richter G, Ma D, Ribeiro RM, Xu C, Cornell E, Stock JL, He T, Mobley AD, Ross S, et al: Early microbial translocation blockade reduces SIV-mediated inflammation and viral replication. J Clin Invest 124: 2802-2806, 2014.

10. Smith AJ, Schacker TW, Reilly CS and Haase AT: A role for syndecan 1 and claudin 2 in microbial translocation during HIV 1 infection. J Acquir Immune Defic Syndr 55: 306-315, 2010.

11. Cicala C, Arthos J and Fauci AS: Role of T-cell trafficking in the pathogenesis of HIV disease. Curr Opin HIV AIDS 14: 115-120, 2019.

12. Jenabian MA, El-Far M, Vyboh K, Kema I, Costiniuk CT, Thomas R, Baril JG, LeBlanc R, Kanagaratham C, Radzioch D, et al; Montreal Primary infection and Slow Progressor Study Groups: Immunosuppressive tryptophan catabolism and gut mucosal dysfunction following early HIV infection. J Infect Dis 212: 355-366, 2015.

13. Sharp PM and Hahn BH: Origins of HIV and the AIDS pandemic. Cold Spring Harb Perspect Med 1: a006841, 2011.

14. Faria NR, Rambaut A, Suchard MA, Baele G, Bedford T, Ward MJ, Tatem AJ, Sousa JD, Arinaminpathy N, Pépin J, et al: HIV epidemiology. The early spread and epidemic ignition of HIV-1 in human populations. Science 346: 56-61, 2014.

15. Worobey M, Watts TD, McKay RA, Suchard MA, Granade T, Teuwen DE, Koblin BA, Heneine W, Lemey P and Jaffe HW: 1970s and 'Patient 0' HIV-1 genomes illuminate early HIV/AIDS history in North America. Nature 539: 98-101, 2016

16. Marchetti G, Tincati C and Silvestri G: Microbial translocation in the pathogenesis of HIV infection and AIDS. Clin Microbiol Rev 26: 2-18, 2013.

17. Lu TX and Rothenberg ME: MicroRNA. J Allergy Clin Immunol 141: 1202-1207, 2018.

18. Yang Y, Ma Y, Shi C, Chen H, Zhang H, Chen N, Zhang P, Wang F, Yang J, Yang J, et al: Overexpression of miR-21 in patients with ulcerative colitis impairs intestinal epithelial barrier function through targeting the Rho GTPase RhoB. Biochem Biophys Res Commun 434: 746-752, 2013
19. Zhao Y, Ma T, Chen W, Chen Y, Li M, Ren L, Chen J, Cao R, Feng Y, Zhang H, et al: MicroRNA-124 promotes intestinal inflammation by targeting aryl hydrocarbon receptor in Crohn's Disease. J Crohn's Colitis 10: 703-712, 2016.

20. Chassin C, Kocur M, Pott J, Duerr CU, Gütle D, Lotz M and Hornef MW: miR-146a mediates protective innate immune tolerance in the neonate intestine. Cell Host Microbe 8: 358-368, 2010.

21. Huang J, Wang F, Argyris E, Chen K, Liang Z, Tian H, Huang W, Squires K, Verlinghieri $G$ and Zhang H: Cellular microRNAs contribute to HIV-1 latency in resting primary CD4+ T lymphocytes. Nat Med 13: 1241-1247, 2007.

22. Xu Y, Wang HW, Luo HY, Shu R, Liu J, Sun L, Han XF, Lin N, Wang TH, Zeng YJ, et al: MicroRNA expression profiling of intestinal mucosa tissue predicts multiple crucial regulatory molecules and signaling pathways for gut barrier dysfunction of AIDS patients. Mol Med Rep 16: 8854-8862, 2017.

23. Ducroc R, Sakar Y, Fanjul C, Barber A, Bado A and Lostao MP: Luminal leptin inhibits L-glutamine transport in rat small intestine: Involvement of ASCT2 and B0AT1. Am J Physiol Gastrointest Liver Physiol 299: G179-G185, 2010.

24. Akgün Şahin Z and Dayapoğlu N: Effect of progressive relaxation exercises on fatigue and sleep quality in patients with chronic obstructive lung disease (COPD). Complement Ther Clin Pract 21: 277-281, 2015.

25. Kekuda R, Prasad PD, Fei YJ, Torres-Zamorano V, Sinha S, Yang-Feng TL, Leibach FH and Ganapathy V: Cloning of the sodium-dependent, broad-scope, neutral amino acid transporter Bo from a human placental choriocarcinoma cell line. J Biol Chem 271: 18657-18661, 1996.

26. van Geldermalsen M, Wang Q, Nagarajah R, Marshall AD, Thoeng A, Gao D, Ritchie W, Feng Y, Bailey CG, Deng N, et al: ASCT2/SLC1A5 controls glutamine uptake and tumour growth in triple-negative basal-like breast cancer. Oncogene 35: 3201-3208, 2016

27. Cai C, Zeng B, Zeng J, Xin H and Tang C: Effect of ASCT2 gene knock-down by shRNA on biological behaviors of colorectal cancer cells. Zhonghua Wei Chang Wai Ke Za Zhi 20: 450-454, 2017 (In Chinese).

28. Sun HW, Yu XJ, Wu WC, Chen J, Shi M, Zheng L and Xu J: GLUT1 and ASCT2 as predictors for prognosis of hepatocellular carcinoma. PLoS One 11: e0168907, 2016.

29. Kasai N, Sasakawa A, Hosomi K, Poh TW, Chua BL, Yong WP, So J, Chan SL, Soong R, Kono K, et al: Anti-tumor efficacy evaluation of a novel monoclonal antibody targeting neutral amino acid transporter ASCT2 using patient-derived xenograft mouse models of gastric cancer. Am J Transl Res 9: 3399-3410, 2017.

30. Guo H, Xu Y, Wang F, Shen Z, Tuo X, Qian H, Wang H and Wang K: Clinical associations between ASCT2 and p mTOR in the pathogenesis and prognosis of epithelial ovarian cancer. Oncol Rep 40: 3725-3733, 2018.

31. Aids group Soid: Chinese medical association: Guideline of diagnosis and treatment for aids. Chin J Infect Dis 29: 629-640, 2011.

32. Livak KJ and Schmittgen TD: Analysis of relative gene expression data using real-time quantitative PCR and the 2(-Delta Delta C(T)) Method. Methods 25: 402-408, 2001.

33. Agarwal V, Bell GW, Nam JW and Bartel DP: Predicting effective microRNA target sites in mammalian mRNAs. eLife 4: 4, 2015

34. Rhoads JM, Niu X, Odle J and Graves LM: Role of mTOR signaling in intestinal cell migration. Am J Physiol Gastrointest Liver Physiol 291: G510-G517, 2006.

35. Sampson LL, Davis AK, Grogg MW and Zheng Y: mTOR disruption causes intestinal epithelial cell defects and intestinal atrophy postinjury in mice. FASEB J 30: 1263-1275, 2016.

36. Duprez DA, Kuller LH, Tracy R, Otvos J, Cooper DA, Hoy J, Neuhaus J, Paton NI, Friis-Moller N, Lampe F, et al; INSIGHT SMART Study Group: Lipoprotein particle subclasses, cardiovascular disease and HIV infection. Atherosclerosis 207: 524-529, 2009.

37. Aqil M, Mallik S, Bandyopadhyay S, Maulik U and Jameel S: Transcriptomic analysis of mRNAs in human monocytic cells expressing the HIV-1 Nef protein and their exosomes. BioMed Res Int 2015: 492395, 2015.

38. Estes JD, Harris LD, Klatt NR, Tabb B, Pittaluga S, Paiardini M, Barclay GR, Smedley J, Pung R, Oliveira KM, et al: Damaged intestinal epithelial integrity linked to microbial translocation in pathogenic simian immunodeficiency virus infections. PLoS Pathog 6: e1001052, 2010. 
39. Tili E, Michaille JJ, Piurowski V, Rigot B and Croce CM: MicroRNAs in intestinal barrier function, inflammatory bowel disease and related cancers-their effects and therapeutic potentials. Curr Opin Pharmacol 37: 142-150, 2017.

40. Poltronieri P, Sun B and Mallardo M: RNA Viruses: RNA roles in pathogenesis, coreplication and viral load. Curr Genomics 16: 327-335, 2015.

41. Ye D, Guo S, Al-Sadi R and Ma TY: MicroRNA regulation of intestinal epithelial tight junction permeability. Gastroenterology 141: 1323-1333, 2011.

42. Vongrad V, Imig J, Mohammadi P, Kishore S, Jaskiewicz L, Hall J, Günthard HF, Beerenwinkel N and Metzner KJ: HIV-1 RNAs are not part of the Argonaute 2 associated RNA interference pathway in macrophages. PLoS One 10: e0132127, 2015.

43. Zou T, Rao JN, Liu L, Xiao L, Chung HK, Li Y, Chen G, Gorospe $M$ and Wang JY: JunD enhances miR-29b levels transcriptionally and posttranscriptionally to inhibit proliferation of intestinal epithelial cells. Am J Physiol Cell Physiol 308: C813-C824, 2015.
44. Hassanein M, Hoeksema MD, Shiota M, Qian J, Harris BK, Chen H, Clark JE, Alborn WE, Eisenberg R and Massion PP: SLC1A5 mediates glutamine transport required for lung cancer cell growth and survival. Clin Cancer Res 19: 560-570, 2013.

45. Chen SD, Chen YB, Peng Y, Xu J, Chen SS, Zhang JL, Li ZZ and Tan Z: Role of PI3K/Akt signaling in the protective effect of magnesium sulfate against ischemia-perfusion injury of small intestine in rats. Chin Med J (Engl) 123: 1447-1452, 2010.

46. Huang Y, Feng Y, Wang Y, Wang P, Wang F and Ren H: Severe burn-induced intestinal epithelial barrier dysfunction is associated with endoplasmic reticulum stress and autophagy in mice. Front Physiol 9: 441, 2018.

(i) () () This work is licensed under a Creative Commons Attribution-NonCommercial-NoDerivatives 4.0 International (CC BY-NC-ND 4.0) License. 